\title{
Finding weight loss strategies that work in primary care
}

The results of two randomized trials shed light on the effectiveness of various weight loss strategies designed for primary care.

The lifestyle intervention in the Diabetes Prevention Program (DPP) clinical study was effective at producing weight loss and reducing the incidence of type 2 diabetes mellitus. In the first study, Ma et al. compared the effectiveness of two DPP lifestyle interventions (adapted for use in primary care) with usual care. The three-arm randomized controlled clinical trial included adults with overweight or obesity and prediabetes and/or the metabolic syndrome who received a coach-led group intervention $(n=79)$, self-directed DVD-based intervention $(n=81)$ or usual care $(n=81)$. The trial had a 3-month intervention phase and a 12-month maintenance phase.

Both of the tested interventions resulted in significant reductions in body weight and waist circumference measurements, and improvements in fasting plasma glucose levels. "This study provides two empirically supported programmes that can be readily adopted into routine practice," concludes lead author Jun Ma of the Palo Alto Medical Foundation Research Institute.

In the second study, Spring and co-workers tested whether mobile technology added to standard-of-care obesity treatment for 69 individuals with overweight or obesity could improve weight loss. In the 6-month intervention phase of the 12-month randomized controlled trial, all participants had biweekly health education classes. Participants in the control group tracked diet and physical activity levels using paper records, whereas those in the experimental group used an app to track and upload their data to a coach. The coach counselled the patients by phone for $10-15$ min every 2 weeks.

Weight loss was significantly greater in the group who used the app than in the group who received standard-of-care treatment alone. However, individuals who used the app only lost weight if they attended the health education classes.

"Technology offers a very effective way to deliver components of weight loss treatment," concludes study researcher Bonnie Spring of

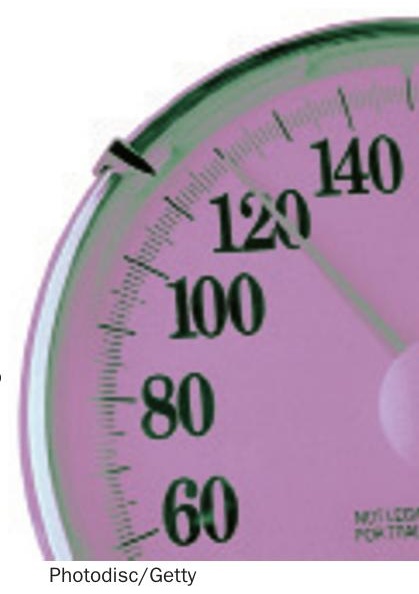
Northwestern University. "By providing feedback and decision support in the moment, it reinforces selfmonitoring. Having a coach track and provide personalized feedback augments the patient's feeling of being supported."

\section{Carol Wilson}

Original articles Ma, J. et al. Translating the Diabetes
Prevention Program lifestyle intervention for weight loss
into primary care: a randomized trial. Arch. Intern. Med.
doi:10.1001/2013.jamainternmed.987 | Spring, B. et al.
Integrating technology into standard weight loss treatment:
a randomized controlled trial. Arch. Intern. Med.
doi:10.1001/jamainternmed.2013.1221

